\title{
Mechanisms for photon sorting based on slit-groove arrays
}

\author{
F. Villate-Guío ${ }^{\mathrm{a}}$, L. Martín-Moreno ${ }^{\mathrm{a}}, \mathrm{F}$. de León-Pérez ${ }^{\mathrm{a}, \mathrm{b}, *}$ \\ ${ }^{a}$ Instituto de Ciencia de Materiales de Aragón and Departamento de Física de la Materia \\ Condensada, CSIC-Universidad de Zaragoza, E-50009 Zaragoza, Spain \\ ${ }^{b}$ Centro Universitario de la Defensa de Zaragoza, Ctra. de Huesca s/n, E-50090 Zaragoza, \\ Spain
}

\begin{abstract}
Mechanisms for one-dimensional photon sorting are theoretically studied in the framework of a coupled-mode method. The considered system is a nanopatterned structure composed of two different pixels drilled on the surface of a thin gold layer. Each pixel consists of a slit-groove array designed to squeeze a large fraction of the incident light into the central slit. The Double-Pixel is optimized to resolve two different frequencies in the near infrared. This system shows high transmission efficiencies and a small crosstalk. It is found that the response of the system strongly depends on the effective area shared by overlapping pixels. According to such degree of overlap, photon sorting can be achieved within three different regimes, which are discussed in detail. Optimal photon-sorting efficiencies are obtained for a moderate number of grooves that overlap with grooves of the neighbor pixel. These results could be applied to both optical and infrared detectors.

Keywords: photon sensing, photon sorting, nanostructured metals, plasmonics
\end{abstract}

\section{Introduction}

Coupling between electromagnetic fields and surface modes in patterned metallic nanolayers offers the possibility for new mechanisms to guide, trap

\footnotetext{
${ }^{*}$ Corresponding author

Email address: fdlp@unizar.es (F. de León-Pérez)
}

Preprint submitted to Photonics and Nanostructures

November 12, 2014 
and localize light [1]. The optical response of nanostructured metallic layers 5 is characterized by narrow spectral bands with resonant wavelengths mainly determined by the periodicity of the structure. Therefore, these systems can be used as filters by just tuning the periodicity [2]. A full analysis of the dependence of such resonances on other geometrical parameters is also available in the literature for systems like hole arrays [3] and apertures surrounded by corrugations $[4,5,6,7,8,9,10,11,12,13,14]$.

In technological applications, like digital cameras or displays, color discrimination is performed through arrays of pixels, where each pixel acts as a separate entity sensitive to a single color [15]. Multispectral sensitivity have also been demonstrated in systems like waveguide resonators $[16,17,18]$ and light har15 vesting structures; for instance, in triple bull's eye structures and triangular lattices of slit-groove arrays (SGAs) [19], or in a mosaic of free-standing arrays of slits used as band pass filter[20]. Such arrangements of nanostructured metallic pixels with multiple spectral resonances behave as wavelength-selective devices with promising advantages in spatial resolution.

In addition to their capability for selecting frequencies, these devices are also able to guide photons with different wavelengths through different channels, i.e. they can be considered as photon sorters; see, for instance, the overlapping light-collection structures reported in Ref. [19], where each pixel is devoted to harvest light of a single color and squeeze it through the central aperture even 25 from the region where it overlaps with other collectors. Authors of Ref. [19] claim that, if photodetectors would be placed underneath the apertures, such devices could act as a miniature spectrometer that detects different wavelengths in the same area. It can be used to generate an image of the object, fulfilling in this way the requirements of the spectral imaging methodology [21].

Laux et al. have proposed both 2D (a triple bull's eye structures) and 1D (SGAs) versions of such photon sorters [19]. Notice that SGAs with different orientations were also arranged in a 2D triangular lattice making them sensible to both polarization and wavelength . These authors have found that the transmission peak intensity of the sorting device relative to that of the isolated pixel 


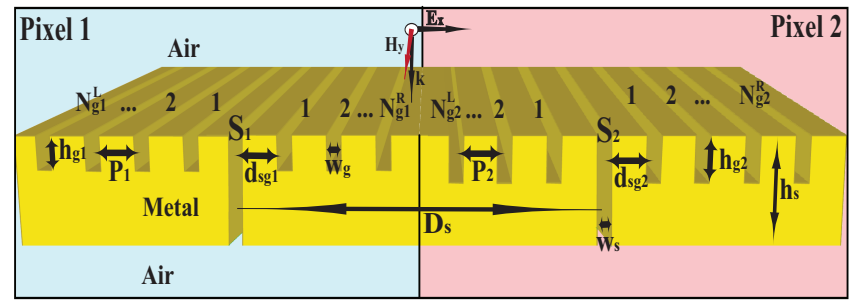

Figure 1: (Color). Schematic representation of the Double-Pixel. Both pixels are sculptured on a uniform gold layer with thickness $h_{s}$. Pixel 1 has a central slit $S_{1}$ of width $w_{s_{1}}$ surrounded by grooves of periodicity $P_{1}$, depth $h_{g_{1}}$ and width $w_{g_{1}}$. The distance between the slit and the nearest groove is $d_{s g_{1}}$. Similar parameters are defined for pixel 2. The distance between slits is $D_{s}$.

drops far more slowly than the percentage spatial overlap between the pixels. That is a signature of the low cross talk between individual pixels. However, one should expect that the interaction between pixels cannot be neglected under general conditions. To the best of our knowledge, the role played by the pixel interaction in photon-sorting devices has not been previously studied. In fact,

40 we show in this paper that the process of photon sorting strongly depends on the pixel overlap and also that it varies in a non-monotonous way.

We choose the simplest version of the 1D photon sorter: two interacting pixels. We study, first, the main physical mechanisms appearing in the process of photon sorting and, second, how to optimize such photon sorters for having an enhanced response.

The building block of the system is a SGA that consists of a thin gold layer (optically opaque) perforated with a subwavelength slit, which is surrounded by an array of periodic grooves sculpted on the illuminated surface, see Fig 1. Geometrical parameters of the SGA are adjusted in order to make the system resonant at a given wavelength. Optimal geometries for a single SGA can be obtained following simple rules recently reported [7, 11, 22].

We first design two isolated SGAs with optimal response at targeted wavelengths $\lambda_{1}$ and $\lambda_{2}$ and then the two SGAs are arranged in the Double-Pixel shown in Fig 1 that resolves both wavelengths with a small cross-talk between 
the pixels. As we are interested in efficient mechanisms to collect the light impinging on a given pixel and redirect it to the other, i.e. to design and implement a photon sorter, we study the change in the optical response of the Double-Pixel as a function of the overlap between the SGAs. The system can be integrated with a standard photodetector $[23,24,25,26,27]$, sensitive to the narrow band of the resonant wavelength.

We focus our attention on the near-infrared part of the spectrum. Integrating light harvesting structures on IR detectors has been recently proposed as an efficient way to increase the absorption of light in a given volume [28]. In this way it is possible to reduce the noise and raise the output signal. Results obtained here can be easily extended to other parts of the IR spectrum as well as to optical frequencies.

The paper is organized as follows. Next section describes the theoretical framework. Our results are discussed in Sec. 3. Section 3.1 first studies the spectral response of non-overlapping pixels. In Sec. 3.2 we evaluate the influence of the overlap between pixels, paying attention to physical mechanisms for photon sorting. The influence of the number of grooves in the optical response of overlapping pixels is analyzed in Sec. 3.3. Our main conclusions are presented at the end of the paper.

\section{Theoretical framework}

A schematic representation of the Double-Pixel is given in Fig. 1. Both pixels are sculptured in a uniform gold layer with thickness $h_{s}$. The dielectric constant of gold is taken from Ref. [29]. Pixel 1 has a central slit $S_{1}$ of width $w_{s_{1}}$ surrounded by grooves of periodicity $P_{1}$, depth $h_{g_{1}}$ and width $w_{g_{1}}$. The distance between the slit and the nearest groove is $d_{s g_{1}}$. Similar parameters are defined for pixel 2. The distance between slits is $D_{s}$. The system is illuminated by a $p$-polarized plane wave, with its electric field parallel to the $\mathrm{x}$ axis, see Fig. 1; the propagation direction is perpendicular to the metal surface. An enhanced transmission is achieved for $p$ polarization [7]. 
The number of grooves at either side of the slit can be different. We define $N_{g_{i}}^{L}, N_{g_{i}}^{R}$ as the number of grooves located at the left and right sides of the slit in the $i$-th pixel, respectively. We use $N_{g_{1}}^{L}=N_{g_{2}}^{R}=6$ along the paper. Only the number of grooves located between the two slits $\left(N_{g_{1}}^{R}\right.$ and $\left.N_{g_{2}}^{L}\right)$ is changed.

Our calculations are performed within the framework of the coupled-mode method (CMM) [1]. This semi-analytical approach nicely reproduces experimental results on SGAs $[26,30,31]$. The CMM is based on a convenient representation of the EM fields. Above and below the metal film the fields are expanded into an infinite set of plane waves with both p- and s-polarizations. Inside slit and grooves the most natural basis is a set of planar waveguide modes [32]. Convergence is fast achieved with a small number of such waveguide modes.

${ }_{95}$ The parallel components of the fields are matched at the metal/air interface using surface impedance boundary conditions [33]. These boundary conditions are also applied at the lateral walls of slit and grooves [34]. After matching the fields at the interface we arrive to a linear system of tight binding-like equations that can be easily solved $[7,35]$.

Using the CMM, we compute the normalized-to-area transmittance $(\eta)$, which is defined as the intensity of the light radiated to the far-field normalized to the intensity of the light incident on the area of the slits. It accounts for the efficiency of the light harvesting process: $\eta$ is of the order of 1 for a single slit, whereas it could become one or two orders of magnitude larger when the groove array squeezes additional light to the central slit $[7,11,22]$.

\section{Mechanisms for the photon sorting}

\subsection{Spectral response}

The largest transmittance for an isolated SGA is obtained when the FabryPerot mode of the slit is located at the same spectral position of the groove cavity mode [7]. For a given wavelength, the spectral position of the FabryPerot mode can be tuned by both metal thickness $h_{s}$ and slit width $w_{s}$, while the groove cavity mode of a groove array is a function of the groove depth and 
width [7]. The optimal periodicity should guarantee that all light re-emitted from the grooves reach the other grooves and the central slit in phase. Varying the distance from the slit to its nearest groove allows a further control of the interaction between the slit and the groove array. Groove pitch and depth are the most relevant design parameters. Ideal values of $w_{g}$ and $d_{s g}$ allow for a finer tuning of the transmittance. Detailed design rules have been reported in a previous work [22].

As a proof of principle, pixels 1 and 2 are designed to operate at $\lambda_{1}=1.35$ $\mu \mathrm{m}$ and $\lambda_{2}=1.50 \mu \mathrm{m}$, respectively. A typical experimental value of $w_{s}=100$ $\mathrm{nm}$ is chosen for the slit width. Both slits have the same width for the sake of simplicity. We use a constant metal thickness $h_{s}=390 \mathrm{~nm}$, which is the arithmetic mean of the optimal thickness values needed to excite the FabryPerot modes in $S_{1}$ and $S_{2}$. This uniform layer does not favor any particular Fabry-Perot mode. The geometry of groove array 1 is $P_{1}=1236 \mathrm{~nm}, h_{g_{1}}=115$ $\mathrm{nm}, w_{g_{1}}=363 \mathrm{~nm}$, and $d_{s g_{1}}=1135 \mathrm{~nm}$, while for the groove array 2 we have $P_{2}=1380 \mathrm{~nm}, h_{g_{2}}=135 \mathrm{~nm}, w_{g_{2}}=363 \mathrm{~nm}$, and $d_{s g_{2}}=1280 \mathrm{~nm}$. Both groove arrays have 6 grooves at each side of the slit. Ref. [22] shows that well-defined and high-intensity transmission peaks with a full-width at halfmaximum (FWHM) of the order of $100 \mathrm{~nm}$ are obtained for a SGA with 12 grooves.

Let us first consider the optical response of a Double-Pixel with a given slit-slit distance $D_{s}=19.4 \mu \mathrm{m}$, so that the constituent SGAs do not overlap. ${ }_{135}$ Fig 2(a) shows the normalized-to-area transmittance $\eta$ as a function of the wavelength.

The Double-Pixel spectrum is compared with those for the isolated pixels 1 and 2, which exhibit narrow well-defined peaks with similar intensities. Peaks of the Double-Pixel are well resolved with a FWHM of about $100 \mathrm{~nm}$ and a cross talk smaller than $1.0 \%$. The crosstalk is defined as the fraction of the total light transmitted by one pixel when only the other pixel is illuminated.

Notice that the transmittance of the Double-Pixel is normalized to the power incident on the total area occupied by both slits. In order to compare the 
Double-Pixel with the isolated pixels on an equal footing, the spectra for the the slits $\left(D_{s}\right)$. This behavior is discussed in the next section.

\subsection{Dependence on the slit-slit distance}

Fig. 3 illustrates the dependence of the transmission spectrum on the slitslit distance $D_{s}$. The contour plot in Fig. 3 (a) represents $\eta$ as a function of both wavelength and $D_{s}$. On the top axis is also given the percentage spatial overlap, which is defined as

$$
\Lambda\left(D_{s}\right)=\left(L_{1}+L_{2}-2 D_{s}\right) /\left(L_{1}+L_{2}+2 D_{s}\right) \times 100 \%
$$

for $L_{1}+L_{2}>2 D_{s}$ and 0 otherwise; where $L_{1}$ and $L_{2}$ are the sizes of pixel 1 and 2, respectively. Crosscuts at $\lambda_{1}=1.35 \mu \mathrm{m}$ and $\lambda_{2}=1.50 \mu \mathrm{m}$ are shown in Fig. 3 (b) and (c), respectively.

We find three regimes with markedly different optical responses. They differ in the effective area shared by the overlapping pixels: 
i) In regime I (RI), the two pixels do not overlap, $\Lambda\left(D_{s}\right)=0$. It occurs in Fig. 3 for $D s \geq\left(L_{1}+L_{2}\right) / 2=15.7 \mu \mathrm{m}$. used above in Fig. 2(a), is between the two local maxima for $\lambda_{1}$ and $\lambda_{2}$, in 
order to obtain similar intensities for the double-peak in the Double-Pixel. Such behavior is related to the different physical origin of the peaks, which is even more apparent in Figs. 3 (b) and (c).

First, we keep $\lambda_{1}=1.35 \mu \mathrm{m}$ as constant and let $D_{s}$ vary, as shown in Fig. 3 (b). In order to explain the optical response of the Double-Pixel, we compare it with two simpler systems: a Double-Slit (DS) for which all grooves are removed and only a single slit remains in each pixel (see [6, 36, 37, 38, 39, 40, 41, 42] for a full discussion of its optical response), and the Pixel-Slit (PS) structure, where the grooves of one pixel are removed leaving only a single slit, while the other pixel is not changed. A schematic representation of the three systems is given in the inset of Fig. 3 (b).

The main peaks of the three systems are at the same spectral positions, c.f. Double-Pixel, Pixel-Slit and Double-Slit curves in RI of Fig. 3 (b). Therefore, the origin of such peaks can be attributed to the interaction between the two slits as in the simplest Double-Slit structure. However, the intensity of the peaks for both the Double-Pixel and the Pixel-Slit is 5 times larger than that corresponding to the Double-Slit configuration. This is due to the presence of the groove array in pixel 1 that acts like an antenna coupling incident light into surface modes, which squeeze the EM energy into the central aperture of this pixel.

Moreover, the interaction of the SGA in pixel 1 and the single slit in pixel 2 of the Pixel-Slit provides practically the same intensity than for the Double220 Pixel, c.f. red-dashed and black-solid lines in Fig. 3 (b). Thus, the interaction with the groove array of pixel 2 can be, in principle, neglected. In the Pixel-Slit spectrum we observe small secondary peaks due to the interaction of the slits with the groove array in pixel 1. Such peaks are transformed into either small shoulders or asymmetric peaks in the Double-Pixel.

225 In contrast, the peaks of the Double-Pixel that are excited at $\lambda_{2}=1.50$ $\mu \mathrm{m}$ can be related with secondary peaks of the Pixel-Slit, see RI in Fig. 3 (c). Therefore, the interaction between the two groove arrays cannot be neglected in this case. 
Such different trends in Figs. 3 (b) and (c) can be better understood looking back at the analysis of Fig. 2(a). We recall that the weak interaction between the two pixels at $\lambda_{1}$ is related to the minimum in the spectra of pixel 2 at this wavelength, while the optical interaction between the two pixels at $\lambda_{2}$ is due to the tail in the peak of pixel 1.

In order confirm our predictions, we have reduced in $40 \mathrm{~nm}$ the distance between grooves in pixel 1 (originally optimized at $\lambda_{1}=1.35 \mu \mathrm{m}$ ), increasing in this way the spectral separation between the two peaks, and observed that peaks of the Double-Pixel excited at $\lambda_{2}=1.50 \mu \mathrm{m}$ moves to values of $D_{s}$ at which the main peaks of the Pixel-Slit are excited, as in Fig. 3 (b), (such calculations are not shown in the paper). The behavior of the secondary peaks becomes more relevant in both regimes II and III, which will be described in what follows.

As the two pixels approach each other and the groove arrays overlap, their stronger interaction produces an "anticrossing" of the two resonances, see Fig. 3 (a). The nearest spectral separation between the two peaks is found at the boundary between RII and RIII. The effect of the anticrossing in RII is that the highest intensities are no longer at the targeted wavelengths $\lambda_{1}=1.35 \mu \mathrm{m}$ and $\lambda_{2}=1.50 \mu \mathrm{m}$. The peak at $\lambda_{1}$ is red shifted when the distance between the slits is reduced, while the peak at $\lambda_{2}$ is blue shifted. Peaks became also narrower than in RI and their relative intensities change so that peaks at $\lambda_{1}$ have lower intensities than those at $\lambda_{2}$.

By keeping a constant value of $1.35 \mu \mathrm{m}$ for $\lambda_{1}$ as in RII of Fig. 3 (b), we observe not only the aforementioned reduction of the transmitted intensity, but also a departure of the Double-Pixel response from the behavior associated to a Pixel-Slit. Furthermore, secondary peaks of the Pixel-Slit (related with the slit-groove interaction) becomes more relevant for the Double-Pixel, while peaks related to the slit-slit interaction in the Pixel-Slit are strongly suppressed by the new conditions of interference. Such effects become more pronounced as the slits approach each other. Similar features are found for $\lambda_{2}=1.50 \mu \mathrm{m}$, see Fig. 3 (c). The main difference with Fig. 3 (b) is that secondary peaks have been already excited in RI and only become better defined in RII, though their 
ensities also decrease for the presence of the anticrossing.

Additional minima appear when grooves of different pixels are located at the same region of the space, see for instance the zoom of the interval demarcated by a red square in Fig. 3 (b), where more than $60 \%$ of the grooves of one pixel overlap with grooves of the other pixel.

265 onances move away from the anticrossing point and the intensity of the transmission peaks starts to raise. Peaks in RIII become narrower and better defined than in RII. In particular, the peak at $D_{s}=4.66 \mu \mathrm{m}$ for $\lambda_{2}=1.50 \mu \mathrm{m}$ has an intensity larger than for the isolated SGA (represented with a horizontal line) and it practically reaches the intensity of the peaks in RI. In addition, the intensity of this peak is 14 times larger than at the nearest dip. An enhancement of 3 is obtained for the highest intensity peak at $\lambda_{1}=1.35 \mu \mathrm{m}$. Such strong oscillations of $\eta$ should be taken into account when the optimal value of $D_{s}$ is chosen. As the system in RIII covers an area smaller than in RI, its hightransmission peaks decreases again when the spatial overlapping is larger than $60 \%$, in agreement with experimental results of Ref. [19].

When the slit is located at the region corresponding to the grooves of the neighbor pixel, we find that the slit transmits additional light, see for instance

\subsection{Influence of the number of grooves}

It is also worth to study the photon sorting as a function of the number of grooves. We start with the Double-Pixel already optimized in Fig. 3, which has 12 grooves in each pixel. Our goal is to increase the number of grooves that redirect additional photons through the apertures, but keeping fixed the total 

to the right of pixel 1 or to the left of pixel 2, see Fig. 1.

As a proof of principle of the photon sorting, we consider first the situation in which only grooves with the same geometrical parameters as in pixel 2 are added to the left of this pixel. The two SGAs are separated a distance $D_{s}=18.7 \mu \mathrm{m}$, the transmittance peaks as a function of the additional grooves is represented in Fig. 4(a). We find an enhancement of the intensity of the the peak at $\lambda=1.5$ $\mu \mathrm{m}$ and a concomitant reduction of the intensity at $\lambda=1.35 \mu \mathrm{m}$.

Taking also into account that the two targeted wavelengths are excited at different values of $D_{s}$, see Fig. 3, we conclude that is not possible to simultaneously enhance the efficiency of photon sorting for both kinds of photons. According to our calculations, this physical constrain cannot be overcome even by optimizing each additional groove independently or by implementing chirped groove arrays as in Ref. [19].

As a rule of thumb, we suggest to use a moderate number of additional grooves. A typical case is illustrated in Fig. 4 (b), where the two pixels are separated the same distance $D_{s}=19.4 \mu \mathrm{m}$ than in Fig. 2. We observe a systematic reduction of $\eta$ with $\Delta N_{g}$ and local maxima for different number of additional grooves $\left(\Delta N_{g}=5\right.$ and $\Delta N_{g}=9$ for $\lambda_{1}=1.35 \mu \mathrm{m}$ and $\lambda_{2}=1.50$ $\Delta N_{g} \leq 7$. Notice that, despite this reduction in intensity, the overlap of the two pixels is still convenient for practical applications due to the reduction of the total size of the system, as already pointed out in Ref. [19].

\section{Conclusions}

We have studied the processes of sensing and sorting photons with different wavelengths by a Double-Pixel. Each pixel consists of a slit-groove array optimized to harvest light of a given wavelength. The considered wavelengths were $\lambda_{1}=1.35 \mu \mathrm{m}$ and $\lambda_{1}=1.50 \mu \mathrm{m}$. 
We find that the optical interaction between the slit-groove arrays strongly Innovation project MAT2011-28581-C02-02, and the CUD project Ref. 2013-13. We thank F. López-Tejeira for a critical reading of the manuscript.

\section{References}

[1] F. J. García-Vidal, L. Martín-Moreno, T. W. Ebbesen, L. Kuipers, Rev. Mod. Phys. 82 (2010) 729-787. 
[2] C. Genet, T. W. Ebbesen, Nature 445 (2007) 39.

[3] T. W. Ebbesen, H. J. Lezec, H. F. Ghaemi, T. Thio, P. A. Wolff, Nature $391(1998) 667$.

[4] T. Thio, K. M. Pellegrin, R. A. Linke, H. J. Lezec, T. W. Ebbesen, Optics Letters 26 (2001) 1972-1974.

[5] H. J. Lezec, A. Degiron, E. Devaux, R. A. Linke, L. Martín-Moreno, F. J. García-Vidal, T. Ebessen, Science 297 (2002) 820-822.

[6] A. P. Hibbins, J. R. Sambles, C. R. Lawrence, Appl. Phys. Lett. 81 (2002) 4661-4663.

[7] F. J. García-Vidal, H. J. Lezec, T. W. Ebbesen, L. Martín-Moreno, Phys. Rev. Lett. 90 (2003) 213901.

[8] S. Akarca-Biyikli, I. Bulu, E. Ozbay, Appl. Phys. Lett. 85 (2004) 1098-1100.

[9] D. Thomas, H. Hughes, Solid State Communications 129 (2004) 519 - 524.

[10] J. Bravo-Abad, L. Martín-Moreno, F. J. García-Vidal, Phys. Rev. E 69 (2004) 026601.

[11] O. T. A. Janssen, H. P. Urbach, G. W. Hooft, Phys. Rev. Lett. 99 (2007) 043902.

[12] H. W. Kihm, K. G. Lee, D. S. Kim, J. H. Kang, Q.-H. Park, App. Phys. Lett. 92 (2008) 051115.

[13] S. B. Choi, D. J. Park, Y. K. Jeong, Y. C. Yun, M. S. Jeong, C. C. Byeon, J. H. Kang, Q.-H. Park, D. S. Kim, App. Phys. Lett. 94 (2009) 063115.

[14] Z. Wang, M. Zhang, J. Wang, F. Lu, K. Li, A. Xu, App. Phys. Lett. 101 (2012) 061107.

370 [15] B. E. Bayer, Colour imaging array, US patent 3,971,065, 1976.

[16] Z. Kang, G. P. Wang, Opt. Express 16 (2008) 7680. 
[17] K. Diest, J. A. Dionne, M. Spain, H. A. Atwater, Nano Lett. 9 (2009) $2579-2583$.

[18] T. Xu, Y.-K. Wu, X. Luo, L. J. Guo, Nat. Commun. 1 (2010) 59.

${ }_{375}$ [19] E. Laux, C. Genet, T. Skauli, T. W. Ebbesen, Nature Photonics 2 (2008) $161-164$.

[20] R. Haïdar, G. Vincent, S. Collin, N. Bardou, App. Phys. Lett. (2010) 221104.

[21] Y. Garini, I. T. Young, G. McNamara, Cytometry 69A (2006) 735-747.

380 [22] F. Villate-Guío, F. López-Tejeira, F. J. García-Vidal, L. Martín-Moreno, F. de León-Pérez, Opt. Express 20 (2012) 25441-25453.

[23] S. Collin, F. Pardo, J.-L. Pelouard, App. Phys. Lett. 83 (2003) 1521-1523.

[24] T. Ishi, J. Fujikata, K. Makita, T. Baba, K. Ohashi, Japanese Journal of Applied Physics 44 (2005) L364-L366.

${ }_{385}$ [25] Z. Yu, G. Veronis, S. Fan, M. L. Brongersma, Appl. Phys. Lett. 89 (2006) 151116.

[26] L. A. Dunbar, M. Guillaumée, F. de León-Pérez, C. Santschi, E. Grenet, R. Eckert, F. López-Tejeira, F. J. García-Vidal, L. Martín-Moreno, R. P. Stanley, Appl. Phys. Lett. 95 (2009) 011113.

390 [27] P. Berini, Laser Photonics Reviews 8 (2014) 197-220.

[28] R. Stanley, Nature Photonics 6 (2012) 409-411.

[29] E. D. Palik, Handbook of Optical Constants of Solids, Academic, London, 1985.

[30] F. López-Tejeira, S. G. Rodrigo, L. Martín-Moreno, F. J. García-Vidal, E. D. T. W. Ebbesen, J. R. Krenn, I. P. Radko, S. I. Bozhevolnyi, M. U. G. andJ. C. Weeber, A. Dereux, Nat. Phys. 3 (2007) 324. 
[31] F. López-Tejeira, S. G. Rodrigo, L. Martín-Moreno, F. J. García-Vidal, E. Devaux, J. Dintinger, T. W. Ebbesen, J. R. Krenn, I. P. Radko, S. I. Bozhevolnyi, M. U. González, J. C. Weeber, A. Dereux, New J. Phys. 10 (2008) 033035.

[32] J. A. Stratton, Electromagnetic theory, McGraw-Hill, New York, 1941.

[33] J. D. Jackson, Classical electrodynamics, 3 ed., John Wiley, New York, 1999.

[34] H. Lochbihler, et al., J. Mod. Opt. 40 (1993) 1273.

[35] F. de León-Pérez, G. Brucoli, F. J. García-Vidal, L. Martín-Moreno, New J. Phys. 10 (2008) 105017.

[36] C. Sönnichsen, A. C. Duch, G. Steininger, M. Koch, G. von Plessen, J. Feldmann, Appl. Phys. Lett. 76 (2000) 140-142.

[37] H. F. Schouten, N. Kuzmin, G. Dubois, T. D. Visser, G. Gbur, P. F. A. Alkemade, H. Blok, G. W. t Hooft, D. Lenstra, E. R. Eliel, Phys. Rev. Lett. 94 (2005) 053901.

[38] P. Lalanne, J. P. Hugonin, C. Rodier, Phys. Rev. Lett. 95 (2005) 263902.

[39] Y. Alarverdyan, B. Sepúlveda, L. Eurenius, E. Olsson, M. Käll, Nature Physics 3 (2007) 884-889.

${ }_{415}[40]$ D. Pacifici, H. J. Lezec, L. A. Sweatlock, R. J. Walters, H. A. Atwater, Opt. Express 16 (2008) 9222.

[41] F. de León-Pérez, F. J. García-Vidal, L. Martín-Moreno, Phys. Rev. B 84 (2011) 125414.

[42] V. Häfele, F. de León-Pérez, A. Hohenau, L. Martín-Moreno, H. Plank, J. R. Krenn, A. Leitner, Appl. Phys. Lett. 101 (2012) 201102. 

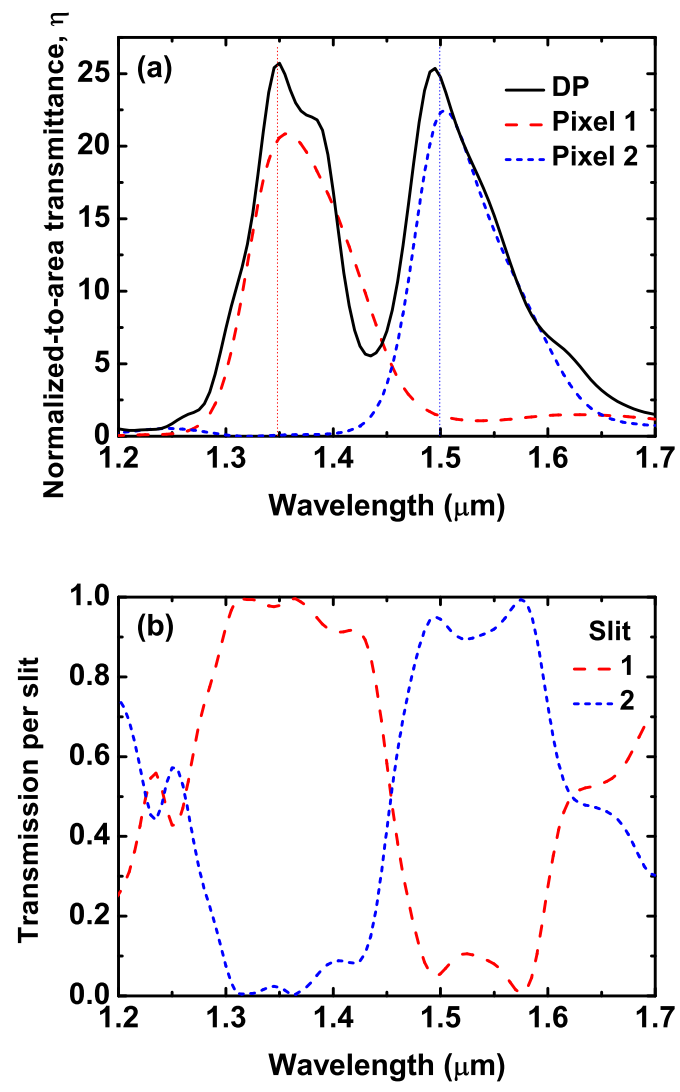

Figure 2: (Color online). (a) Normalized-to-area transmittance $(\eta)$ as a function of wavelength for the Double-Pixel (black-solid line) and the isolated pixels resonant at $\lambda_{1}=1.35 \mu \mathrm{m}$ (reddashed line) and $\lambda_{2}=1.5 \mu \mathrm{m}$ (blue-dotted line). (b) Transmission per slit. The geometry of groove array 1 is $P_{1}=1236 \mathrm{~nm}, h_{g_{1}}=115 \mathrm{~nm}, w_{g_{1}}=363 \mathrm{~nm}$, and $d_{s g_{1}}=1135 \mathrm{~nm}$, while for the groove array 2 we have $P_{2}=1380 \mathrm{~nm}, h_{g_{2}}=135 \mathrm{~nm}, w_{g_{2}}=363 \mathrm{~nm}$, and $d_{s g_{2}}=1280$ $\mathrm{nm}$. The metal thickness is $h_{s}=390 \mathrm{~nm}$. The width of both slits is $w_{s}=100 \mathrm{~nm}$. The slit-slit distance of the Double-Pixel is $D_{s}=19.4 \mu \mathrm{m}$. 

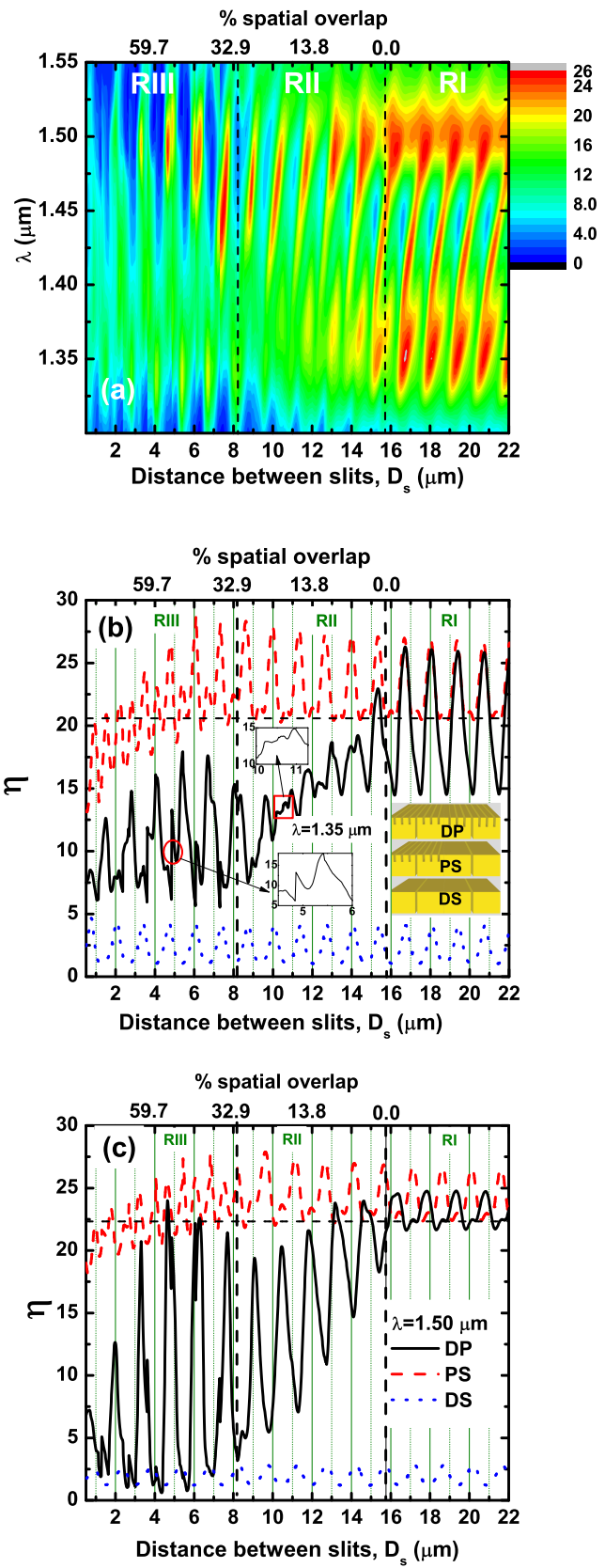

Figure 3: (Color online). (a) Contour plot of $\eta$ for the Double-Pixel as a function of the wavelength $\lambda$ and the slit-slit distance $D_{s}$. The percentage spatial overlap is given on the top axis. (b) Crosscut at $\lambda_{1}=1.35 \mu \mathrm{m}$ for three different systems: i) Double-Pixel (DP, black line), ii) Pixel-Slit (PS, red-dashed line), which consists in pixel 1 and a single slit $S_{2}$, and iii) Double-Slit (DS, blue-pointed line). Schemes of the three structures are shown in the inset. (c) Crosscut at $\lambda_{2}=1.50 \mu \mathrm{m}$ for DP, PS and DS systems. The horizontal dashed lines in (b) and (c) represent the intensity of the isolated SGAs. Geometrical parameters of slit and grooves are the same than in Fig. 2. 

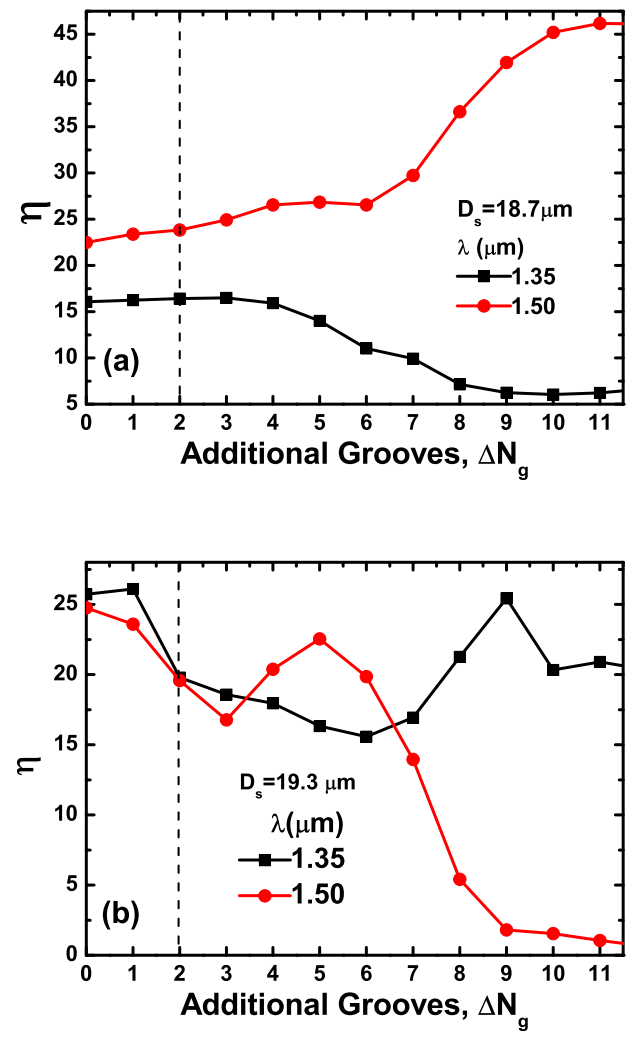

Figure 4: (Color online). Intensity of the transmittance peaks for the two targeted wavelengths $\left(\lambda_{1}=1.35 \mu \mathrm{m}\right.$ and $\left.\lambda_{2}=1.50 \mu \mathrm{m}\right)$ as function of the number of grooves added to: (a) pixel 2 $\left(\Delta N_{g} \equiv N_{g_{2}}^{L}-N_{g_{2}}^{R} \geqq 0\right.$ and $\Delta N_{g_{1}}=N_{g_{1}}^{R}-N_{g_{1}}^{L}=0$, see definition of the structure in Fig. 1) and (b) both pixels $\left(\Delta N_{g} \equiv N_{g_{1}}^{R}-N_{g_{1}}^{L}=N_{g_{2}}^{L}-N_{g_{2}}^{R} \geqq 0\right)$. Geometrical parameters of slits and grooves are the same than in Fig. 2. The vertical line represents the minimal $\Delta N_{g}$ to have overlapping pixels. 